\title{
New perspectives on contributing factors to the monthly behavior of the $a a$ geomagnetic index
}

\author{
Blanca Mendoza ${ }^{1}$, Marni Pazos ${ }^{1 *}$ (1) and Luis Xavier González ${ }^{2}$
}

\begin{abstract}
We studied the Aa geomagnetic index (aa index daily average) behavior on a monthly timescale using data from 1868 to 2015 for cycles 11-24. We identified solar- and lunar-associated periodicities in the Aa time series and found statistically significant Aa minima values a few days before the full Moon and high Aa values during the new Moon. When considering all the cycles, it was clear that the deepest Aa minima occurred during the Aa descending activity phase. However, when the cycles were separated according to the direction of the interplanetary magnetic field (IMF), the Aa minima came from the contribution of cycles with the IMF pointing toward the Sun (Type 1). Furthermore, during the descending phase of cycles with the IMF pointing away from the Sun (Type 2), the smallest Aa index values were found along with smaller changes compared to Type 1 cases. This behavior implies that during Type 1 cycles there are larger Aa perturbations than during Type 2 cycles. It is very likely that the mechanisms responsible for the Aa monthly behavior are a combination of solar and lunar effects that depend on several factors: (a) the Moon phases (new and full Moon), (b) the phase of the solar cycle (ascending or descending), and (c) the direction of the interplanetary magnetic field (away or toward the Sun).
\end{abstract}

Keywords: Moon, Geomagnetic activity, aa index, Solar activity, Interplanetary magnetic field

\section{Background}

It is well known that geomagnetic activity is modulated by solar activity and ionospheric variability produced by lunar and solar tides (Campbell 1997; Richardson et al. 2002; Yamazaki and Richmond 2013; Yamazaki 2014). On a monthly timescale, when a clear lunar role could be expected, the Moon's influence on the geomagnetic activity has been inconclusive. In Michel et al. (1964), the authors studied the correlation between the $K p$ index of geomagnetic activity and 30-year period Moon data phases starting from 1931 and found no correlation. However, using the same data for solar activity cycles 17-19, Stolov and Cameron (1964) found that $K p$ decreases relative to the average value over several days before the full Moon and increases by the same value

\footnotetext{
*Correspondence: marnipazos@gmail.com

${ }^{1}$ Instituto de Geofísica, Universidad Nacional Autónoma de México, 04510 Mexico City, Mexico

Full list of author information is available at the end of the article
}

over several days after the full Moon. In addition, Gulyaev and Gulyaeva (1992) reported increases in the geomagnetic field disturbances during the full Moon at the solar activity minimum using the auroral electrojet index. Střeštík (1998), studying the $A p$ time series, found significant peaks at $\sim 27$ and 13.6 days that he associated with solar rotation and the structure of solar wind. The author claimed that he did not find a peak corresponding to the period of the synodic month (the interval between two successive lunar phases) at 29.5 days or its half. In a recent work, Akimov and Dyatel (2012) showed the dependence of the $A p$ index on the Moon's phase for solar cycles $20-23$ and found that in the declining phase of the solar cycle, the highest values of the $A p$ index relative to an average value are observed near the new Moon. In the ascending and maximum solar cycle phase, they observed minimum values of $A p$ over several days before the full Moon and maximum values of $A p$ for several days after the full Moon. They concluded that the mechanism 
of the Moon's effect on the Earth's magnetosphere is different near the new Moon and full Moon.

In the present study, we identified solar and lunar factors that may contribute to the monthly behavior of geomagnetic activity. To achieve this, we applied the wavelet, fast Fourier transform (FFT), and superposed epoch analysis to the $A a$ geomagnetic index using data from 1868 to the present and applied the superposed epoch analysis to the lunar phases. We further considered the ascending and descending $A a$ index phases, as well as epochs of different direction of the interplanetary magnetic field (IMF), to assess the 11-year solar activity cycle contribution.

\section{Methods}

Geomagnetic disturbances can be monitored by groundbased magnetic observatories recording the three magnetic field components. The $a a$ index is a global geomagnetic activity index derived from the $K$ indices from two approximately antipodal observatories: Hartland in the UK and Canberra in Australia. The $a a$ index was introduced by Mayaud (1972). Since it is based on data from only two observatories, it is the simplest of the three hourly planetary indices. It strongly correlates with the $a p$ and $a m$ indices, which are derived using data from more extensive observatory networks (http://www. geomag.bgs.ac.uk/data_service/data/magneticindices/ aaindex.html). The global $K p$ is a 3 -h index obtained as the mean value of the disturbance levels in the two horizontal field components, observed at 13 selected, sub-auroral observatories. The 3-h $a p$ and the daily $A p$ indices are planetary magnetic activity indices, related to the $K p$ index, and are the average values of the irregular disturbance levels in the horizontal field components observed at selected magnetic observatories worldwide (http://www.gfz-potsdam.de/sektion/erdmagnetfeld/ daten-dienste/kp-index/). The main advantage of using the $a a$ index is that the time series spans from 1868, further back than any of the other planetary index time series. In this study, we used the $a a$ index in order to have a long-term time series between 1868 and 2015. We averaged the $a a$ index to obtain the $A a$ daily index.

In order to analyze local variations of power within a single non-stationary time series at multiple periodicities, we applied the wavelet method using the Morlet wavelet (Torrence and Compo 1998; Grinsted et al. 2004; Mendoza and Velasco 2009). In Fig. 1, we showed the wavelet analysis of the $A a$ time series. We used a high-pass filter with a cutoff frequency set at 64 days. The $A a$ index has significant periodicities at $28 \pm 3$ and $14 \pm 1.5$ days (the uncertainty was obtained from the peak's width at half maximum). Taking into account the uncertainties, these periodicities can be associated with the solar rotation of 27 days, the lunar synodic month of 29.5 days, and their first harmonics. To distinguish the peaks of the $A a$ time series better, we performed a FFT analysis. In Table 1, we presented a summary of the $A a$ cycles 11-24. We identified the first five most prominent peaks within a window of three years, centered in either the cycle's maxima (14 cycles) or minima (13 cycles). Periodicities associated with both the Moon and the Sun or their first and second harmonics appear, although for some cycles we did not find significant peaks.

From Table 1, for each cycle, we obtained the times that the periodicities and/or their harmonics can be related to both the Sun and Moon (S-M), mainly or only to the Moon $(\mathrm{M})$, and mainly or only to the Sun $(\mathrm{S})$. These appeared in Table 2.

Properties of the solar wind change depending on the phase of the 11-year solar activity cycle. The ascending phase and maximum of the solar cycle are dominated by transients such as coronal mass ejections, while the

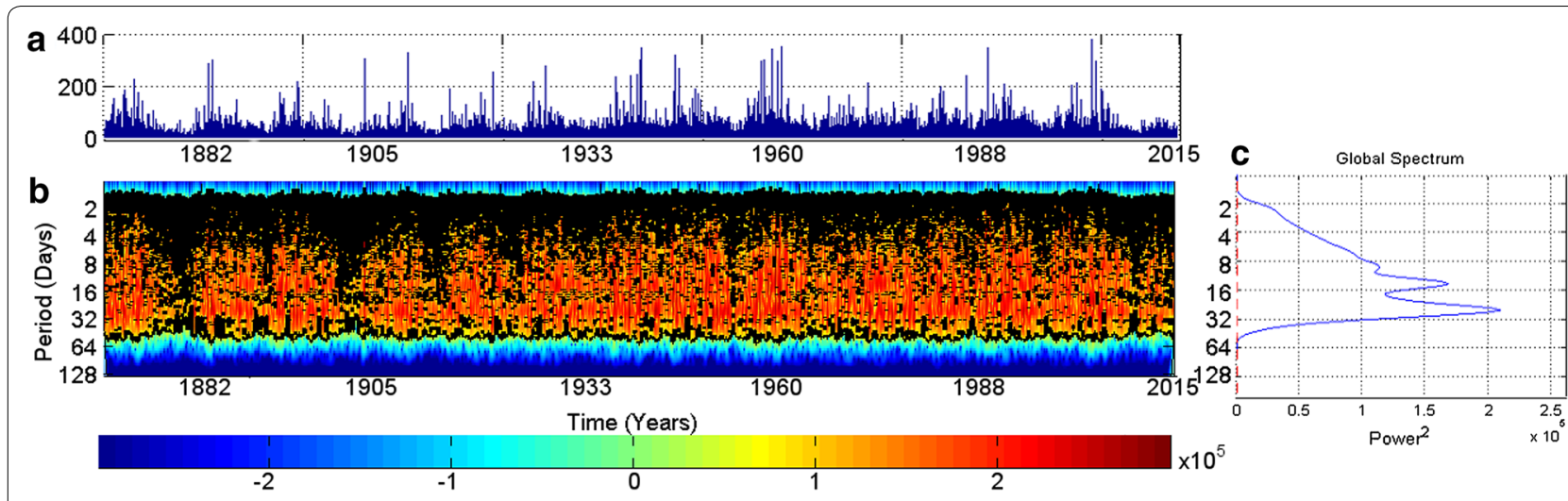

Fig. 1 Wavelet of the Aa geomagnetic index time series. a Aa time series. b Wavelet spectrum. c Global wavelet spectrum. The color code indicating the spectral plot statistical significance level is at the bottom of the figure 
Table 1 Periodicities and harmonics of the $A a$ time series associated with the Moon and the Sun

\begin{tabular}{|c|c|c|c|c|}
\hline Cycle & Maxima (days) & Related to & Minima (days) & Related to \\
\hline 11 & 14.49 & M-1st harmonic & $\begin{array}{l}6.84 \\
6.95\end{array}$ & $\begin{array}{l}S-2 n d \text { harmonic } \\
S-2 n d \text { harmonic }\end{array}$ \\
\hline 12 & - & & 13.61 & S-1st harmonic \\
\hline 13 & $\begin{array}{l}27.51 \\
13.83 \\
13.60\end{array}$ & $\begin{array}{l}\mathrm{S} \\
\mathrm{S}-1 \text { st harmonic } \\
\mathrm{S}-1 \mathrm{st} \text { harmonic }\end{array}$ & $\begin{array}{l}29.02 \\
27.38\end{array}$ & $\begin{array}{l}M \\
S\end{array}$ \\
\hline 14 & $\begin{array}{l}30.18 \\
26.72\end{array}$ & $\begin{array}{l}M \\
S\end{array}$ & $\begin{array}{l}29.02 \\
27.38\end{array}$ & $\begin{array}{l}M \\
S\end{array}$ \\
\hline 15 & 13.45 & S-1st harmonic & $\begin{array}{l}26.74 \\
27.48\end{array}$ & $\begin{array}{l}S \\
S\end{array}$ \\
\hline 16 & 13.61 & $\mathrm{~S}-1 \mathrm{st}$ harmonic & $\begin{array}{l}29.06 \\
13.46\end{array}$ & $\begin{array}{l}\mathrm{M} \\
\mathrm{S}-1 \mathrm{st} \text { harmonic }\end{array}$ \\
\hline 17 & $\begin{array}{l}13.90 \\
27.37\end{array}$ & $\begin{array}{l}\mathrm{S}-1 \text { st harmonic } \\
\mathrm{S}\end{array}$ & - & \\
\hline 18 & $\begin{array}{l}27.27 \\
14.08 \\
29.16\end{array}$ & $\begin{array}{l}\mathrm{S} \\
\mathrm{S}-1 \text { st harmonic } \\
\mathrm{M}\end{array}$ & $\begin{array}{l}26.43 \\
29.65\end{array}$ & $\begin{array}{l}S \\
M\end{array}$ \\
\hline 19 & 13.60 & S-1st harmonic & $\begin{array}{l}31.42 \\
6.94\end{array}$ & $\begin{array}{l}M \\
S\end{array}$ \\
\hline 20 & $\begin{array}{l}13.63 \\
29.89 \\
28.87\end{array}$ & $\begin{array}{l}\mathrm{S}-1 \text { st harmonic } \\
\mathrm{M} \\
\mathrm{M}\end{array}$ & $\begin{array}{l}31.29 \\
14.72\end{array}$ & $\begin{array}{l}M \\
M\end{array}$ \\
\hline 21 & - & & $\begin{array}{l}13.85 \\
15.77\end{array}$ & $\begin{array}{l}S \\
M-1 \text { st harmonic }\end{array}$ \\
\hline 22 & - & & $\begin{array}{l}26.97 \\
15.11\end{array}$ & $\begin{array}{l}S \\
M-1 \text { st harmonic }\end{array}$ \\
\hline 23 & $\begin{array}{l}13.42 \\
27.46\end{array}$ & $\begin{array}{l}\mathrm{S}-1 \mathrm{st} \text { harmonic } \\
\mathrm{S}\end{array}$ & $\begin{array}{l}13.49 \\
27.37\end{array}$ & $\begin{array}{l}\mathrm{S}-1 \mathrm{st} \text { harmonic } \\
\mathrm{S}\end{array}$ \\
\hline 24 & $\begin{array}{l}13.39 \\
28.82\end{array}$ & $\begin{array}{l}\mathrm{S}-1 \text { st harmonic } \\
\mathrm{M}\end{array}$ & & \\
\hline
\end{tabular}

- means that no significant peaks were found

Table 2 Number of periodicities per cycle related to the Sun and the Moon

\begin{tabular}{lll}
\hline & Maxima & Minima \\
\hline S-M & 2 & 7 \\
M & 2 & 1 \\
S & 7 & 4 \\
- & 3 & 1 \\
\hline
\end{tabular}

- means that no significant peaks were found

descending and minimum phases of the solar cycle are dominated by high-speed wind streams (Richardson et al. 2002). These are also the solar sources of geomagnetic activity and of the $A a$ index. Moreover, the IMF changes direction. Epochs corresponding to the ascension of the even numbered solar cycles and the descent of the odd numbered solar cycles are statistically characterized by the IMF pointing toward the Sun. We identified these as Type 1 cycles. The times corresponding to the ascension of the odd cycles and the descent of the even cycles are statistically significant when the IMF is pointing away from the Sun. We identified these as Type 2 cycles. The $A a$ activity index is also influenced by the IMF polarity (Russell and Mulligan 1995). We presented $A a$ Type 1 and 2 cycles in Fig. 2 with the sunspot number time series.

The $A a$ index time series consists of daily data from January 1, 1868, through January 31, 2015, which covers cycles 11 through 24 of the $A a$ index activity. We used the $A a$ cycles and not the sunspot cycles. While both phenomena have a similar time pattern, the time series do not exactly coincide (see Fig. 2). The $a a$ index has several solar-associated sources, and some of them are not related to sunspots. High-speed streams account for approximately two-thirds of the long-term $a a$ averages at solar minimum, while at solar maximum, structures associated with transients (interplanetary coronal mass ejections, shocks, etc.) make the largest contribution ( $50 \%$ ) from streams, and slow solar wind continues to be present. Similarly, high-speed streams are the principal contributor $(\sim 55 \%)$ to solar minimum IMF averages, while transient-related structures are the leading contributor ( 40\%) at solar maximum (Richardson et al. 2002). 

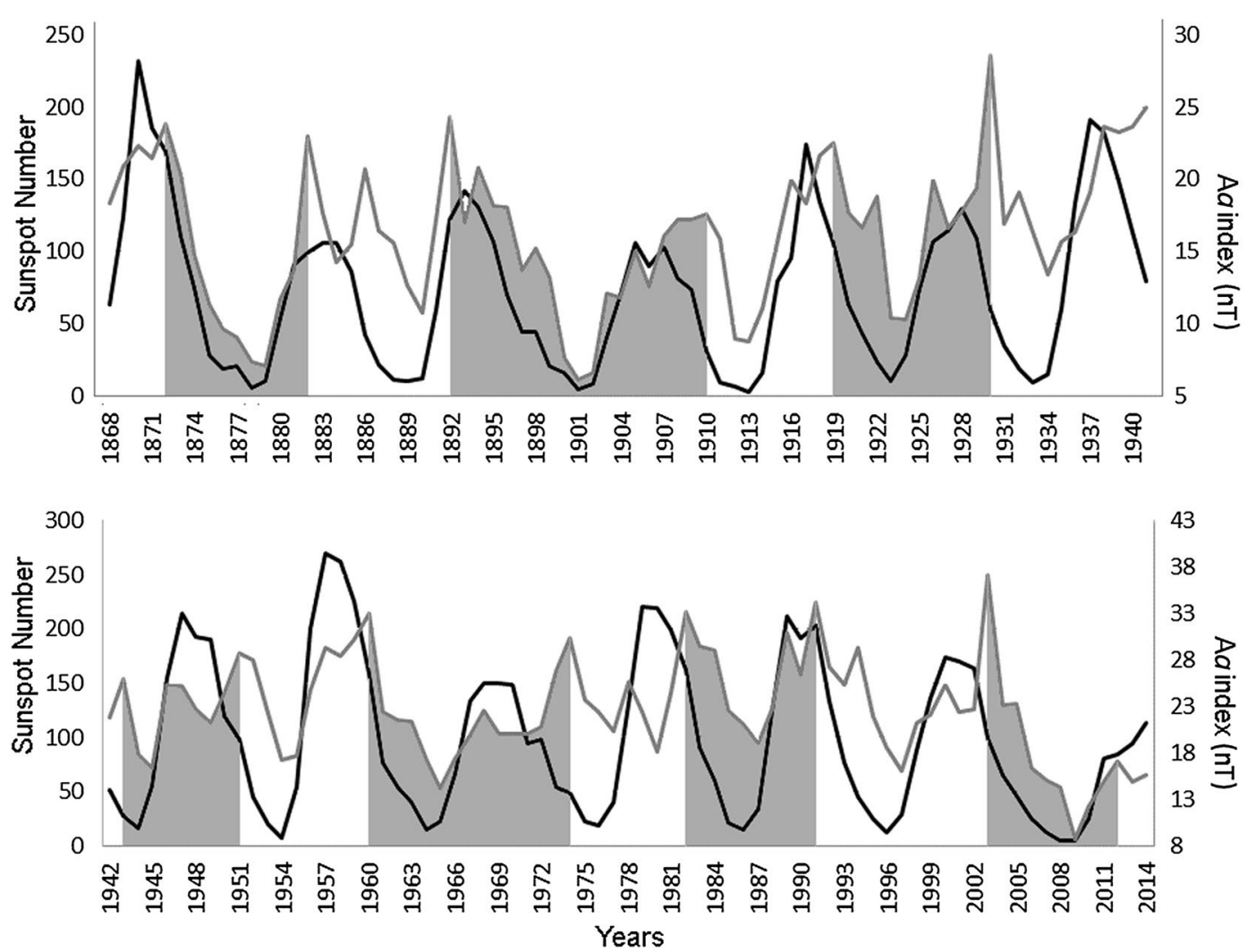

Fig. 2 Time series of the sunspot numbers (black curve) (http://sidc.be/silso/datafiles) and the Aa geomagnetic activity index (gray curve). The gray shadowed areas correspond to Type 1 cycles and the white areas to Type 2 cycles

Table 3 presents the corresponding years of the $A a$ activity cycles $11-24$, as well as the periods of the ascending $(1$ year after the minimum to 1 year before the

Table 3 Years of $\mathrm{Aa}$ cycles and their corresponding ascending and descending phases

\begin{tabular}{llll}
\hline Cycle number & Complete cycles & Ascending phase & $\begin{array}{l}\text { Descending } \\
\text { phase }\end{array}$ \\
\hline 11 & $1868-1879$ & $1868-1872$ & $1872-1879$ \\
12 & $1879-1890$ & $1879-1882$ & $1882-1890$ \\
13 & $1890-1901$ & $1890-1892$ & $1892-1901$ \\
14 & $1901-1913$ & $1901-1910$ & $1910-1913$ \\
15 & $1913-1924$ & $1913-1919$ & $1919-1924$ \\
16 & $1924-1934$ & $1924-1930$ & $1930-1934$ \\
17 & $1934-1945$ & $1934-1943$ & $1943-1945$ \\
18 & $1945-1954$ & $1945-1951$ & $1951-1954$ \\
19 & $1954-1965$ & $1954-1960$ & $1960-1965$ \\
20 & $1965-1980$ & $1965-1974$ & $1974-1980$ \\
21 & $1980-1987$ & $1980-1982$ & $1982-1987$ \\
22 & $1987-1997$ & $1987-1991$ & $1991-1997$ \\
23 & $1997-2009$ & $1997-2003$ & $2003-2009$ \\
24 & $2009-2015$ & $2009-2012$ & $2012-2015$ \\
\hline
\end{tabular}

maximum) and descending (1 year after the maximum to 1 year before the minimum) phases, which were used in the analysis.

From Table 3, we explicitly identified in Table 4 the years of Type 1 and 2 cycles and their corresponding ascending and descending phases.

To construct the 40-day time series presented in Figs. 3 and 4, we proceeded as follows. The first series had only 20 days (January 1-20, 1868), and the full Moon occurred on January 10. The second series had 40 days and spanned from January 10 (full Moon) to February 18, 1868 (the full Moon occurred on February 8). and so on until the end of the time series. These 40-day periods were superposed and averaged in order to obtain the single 40-day series shown in the figures, corresponding to all cycles, cycles Type 1 and 2, and ascending and descending phases. The 40-day period began with the full Moon and ended 10 days after the next full Moon. We adopted this criterion, following Akimov and Dyatel (2012), to observe clearly the behavior of the $A a$ index after the full Moon. The average periods of the new and full Moon are 15 and 30 days, respectively (Additional file 1). 
Table 4 Years of Aa Type 1 and 2 cycles and their corresponding ascending and descending phases

\begin{tabular}{|c|c|c|c|}
\hline \multicolumn{2}{|l|}{ Type 1} & \multicolumn{2}{|l|}{ Type 2} \\
\hline $\begin{array}{l}\text { Descending } \\
\text { phase }\end{array}$ & $\begin{array}{l}\text { Ascending } \\
\text { phase }\end{array}$ & $\begin{array}{l}\text { Descending } \\
\text { phase }\end{array}$ & $\begin{array}{l}\text { Ascending } \\
\text { phase }\end{array}$ \\
\hline 1872-1879 & 1879-1882 & - & 1868-1872 \\
\hline 1892-1901 & 1901-1910 & 1882-1890 & 1890-1892 \\
\hline 1919-1924 & 1924-1930 & 1910-1913 & 1913-1919 \\
\hline 1943-1945 & 1945-1951 & 1930-1934 & 1934-1943 \\
\hline 1960-1965 & 1966-1974 & 1951-1954 & 1954-1960 \\
\hline 1982-1987 & 1987-1991 & 1974-1980 & 1980-1982 \\
\hline \multirow[t]{2}{*}{ 2003-2009 } & 2009-2012 & 1991-1997 & 1997-2003 \\
\hline & & $2012-2015$ & \\
\hline
\end{tabular}

\section{Results}

In Fig. 3, we showed the $A a$ superposed epoch analysis of cycles 11-24 and cycles 20-23. The figure allowed the comparison between the whole period (cycles 11-24) and cycles 20-23 during the ascending and descending phases. The latter is the interval considered by Akimov and Dyatel (2012). The full Moon always occurs after the $A a$ minimum values corresponding to each case. The exception is the descending phase for cycles 20-23 (Fig. 3f), where the full Moon appears during the $A a$ minimum. The new Moon is always observed during periods of high $A a$ values. Figure 4 shows the $A a$ superposed epoch analysis of cycles 11-24 and Type 1 and 2 cycles for the ascending and descending solar cycle phases. In Table 5, we show the calculated results for several $A a$ parameters to quantify the results shown in Fig. 4.

\section{Discussion and conclusions}

The wavelet and FFT analysis suggested that lunar and solar periodicities are mingled. The summary in Table 2 showed several cycles presenting both Sun- and Moonrelated periodicities. In Střeštík (1998), periodicities around 27 days and 29 and 30 days appeared as significant, although the author associated the latter to the modulation by the annual wave in solar tides. Table 2 also shows that during maxima, most periodicities are related to the Sun or mainly the Sun, which is expected since during these epochs the solar activity is higher and should dominate the lunar effects. During minima, the Sun-Moon-related periodicities are more numerous, indicating that the Moon effect is more evident than in times of higher solar activity. Because the lunar cycle and solar rotation have slightly different periods, the relative phase drifts should produce random peaks/dips as shown in Figs. 3 and 4.

Concerning the geomagnetic behavior on monthly timescales, in Akimov and Dyatel (2012) and using the
$A p$ index for cycles 20-23, the authors found the highest values in the declining part of the solar cycle near the new Moon and $a a$ minima near the full Moon. For the ascending and maximum part of the solar cycle, they found minimum values of $A p$ before the full Moon and maximum values after the full Moon. Figure 3d, f shows a behavior qualitatively similar to that described in Akimov and Dyatel (2012). However, we would like to point out that these authors used $A p$ data, while we used $A a$ index data, and, more importantly, they used sunspot cycles, while we use $A a$ index cycles. Therefore, differences in the behavior between the cycles are expected. Figure 3 shows that in all cases there is a strong fall in the $A a$ index value a few days before the full Moon. The full Moon occurs two days after the $A a$ minimum day, and the new Moon appears during high $A a$ value epochs.

In Table 5, we presented several parameters from Fig. 4 and considered statistically significant values those that are equal to or larger than $2 \sigma$. Considering the periods before and after the $A a$ minima, we noticed that the means of the two periods reached similar $A a$ index values (columns two and eight). Concerning the $A a$ minima, the data in the fourth and fifth columns indicate that all the cases present statistically significant minima in the $A a$ index value a few days before the full Moon (see also Fig. 4). The minima occur three days before the full Moon, with two exceptions: the Type 1 descending phase (see Fig. 4f), where the full Moon appears four days after the $A a$ minimum, and the Type 2 descending phase (see Fig. 4i), where it appears six days after the $A a$ minimum. When considering all the cases (ALL, first column), it is clear that the deepest $A a$ minimum occurred during the descending phase (ALL D, first column) according to the number of $\sigma$ s (fifth column). However, when the cycles are separated into Type 1 and 2, the Type 1 descending phase (T1 D) presented the deepest $A a$ minimum, being the main contributor to the ALL D Aa values, while the T2 D presented the smallest minimum (fifth column).

Concerning the new Moon, the data in the sixth and seventh columns show that the associated $A a$ index values are not statistically different from their means in the second column (they are less than $2 \sigma$ ), and, therefore, they presented higher values compared to the $A a$ minima. Nevertheless, the descending phase values are the closest to their averages for all the cases. In addition, during the T1 A and T2 D phases, the $A a$ means were the largest and the smallest, respectively.

Finally, the $A a$ full Moon values were averaged (see column eight). The T2 A phase presented the largest smallest $A a$ average values and T1D and T2D the smallest values, respectively (see columns eight and nine); however, they are not statistically significant (they are less than $2 \sigma$ ). Generally, during the descending phase, 
CYCLES $11-24$
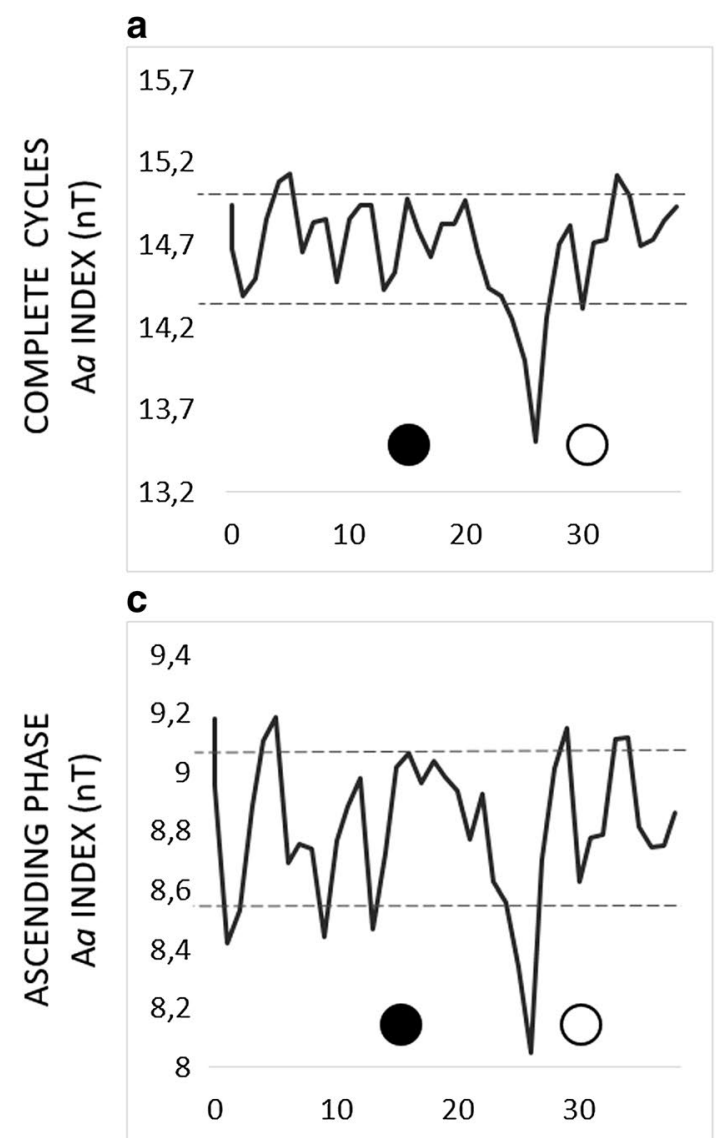

e

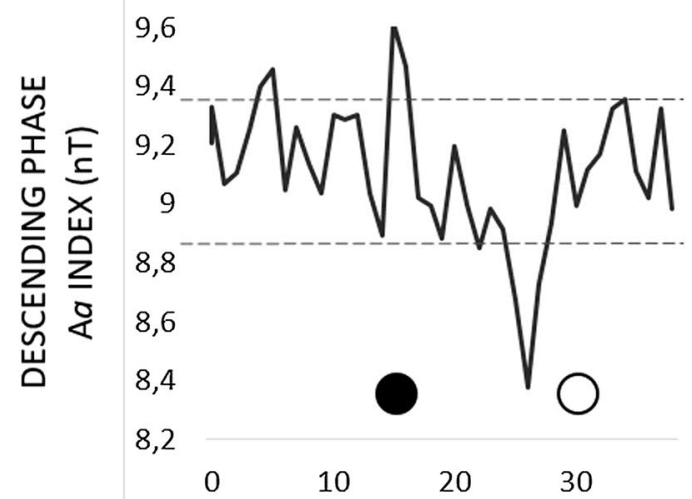

CYCLES $20-23$

b

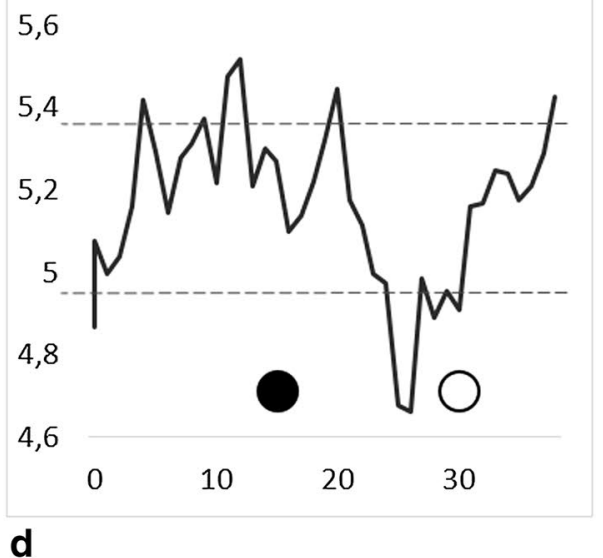

3,2

3,1

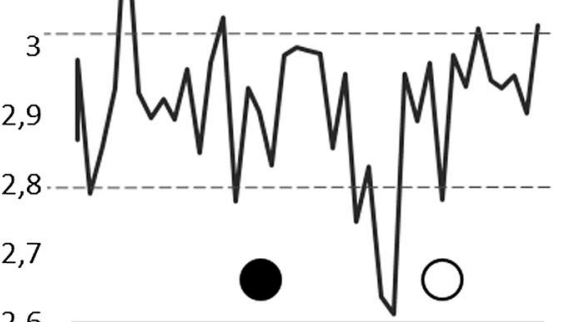

2,6

$\begin{array}{llll}0 & 10 & 20 & 30\end{array}$

f

3,4

3,3

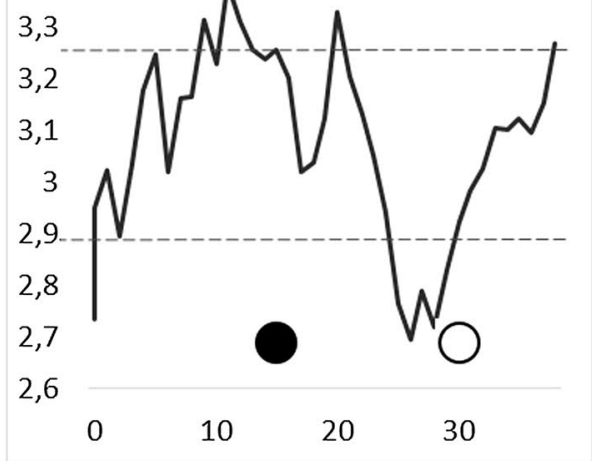

DAYS FROM FULL MOON

Fig. 3 Superposed epoch analysis. Left side Aa index values for cycles 11-24 in composites of 40 days from the full Moon. Right side Aa index values for cycles 20-23 in composites of 40 days from the full Moon. Figures $\mathbf{a}$ and $\mathbf{b}$ correspond to whole cycles, $\mathbf{c}$ and $\mathbf{d}$ to the ascending phase, and $\mathbf{e}$ and $\mathbf{f}$ to the descending phase. The black circles represent the new Moon on the 15th day of the 40-day period. The white circles represent the full Moon at the 30th day of the 40-day period. The horizontal dashed lines correspond to one standard deviation taking the 40-day period; the values for each panel are: a $0.32, \mathbf{b} 0.20, \mathbf{c} 0.25, \mathbf{d} 0.11$, e 0.24 , and $\mathbf{f} 0.18$

the $A a$ values are the smallest and closest to their means (see columns two to nine). In particular, T2 D cycles presented the smallest $A a$ index values of all the cases. Type
1 cases tend to present larger changes than Type 2 cases (see fifth and seventh columns). These two features may indicate that during Type 2 epochs the $A a$ perturbations 


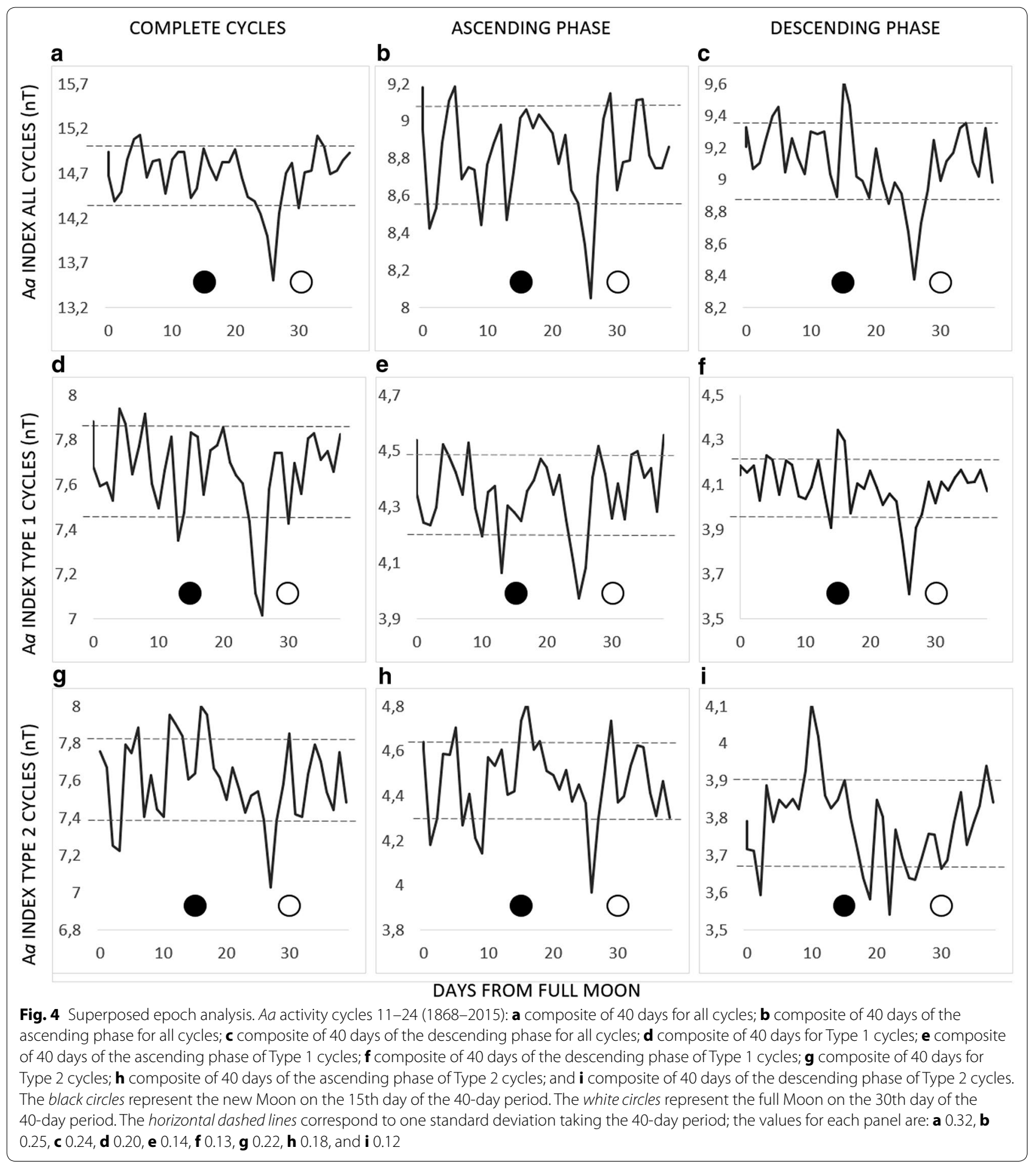

are smaller than during Type 1 epochs. There were A $a$ minima values a few days before the full Moon and higher $A a$ values a few days after.

We looked at possible mechanisms explaining the $A a$ drop around full Moon. Recent observations have established that there is an interaction on magnetically connected field lines of the solar wind and magnetospheric plasma with the Moon and its exosphere, surface, and crustal magnetic fields (e.g., Mitchell et al. 2008; Poppe and Horányi 2010; Saito et al. 2012; Halekas 
Table 5 Values of $A a$ index corresponding to Fig. 4

\begin{tabular}{|c|c|c|c|c|c|c|c|c|}
\hline Cycles & Mean 1-27 & $\sigma$ & aa Min & $\# \sigma$ & New Moon & $\# \sigma$ & Mean 29-40 & $\# \sigma$ \\
\hline ALL & 14.70 & 0.27 & 13.51 & 4.41 & 14.43 & 1.00 & 14.73 & 0.25 \\
\hline ALL A & 8.81 & 0.24 & 8.05 & 3.17 & 8.47 & 1.42 & 8.87 & 0.18 \\
\hline ALLD & 9.14 & 0.22 & 8.38 & 3.50 & 9.04 & 0.50 & 9.11 & 0.19 \\
\hline $\mathrm{T} 1$ & 7.66 & 0.19 & 7.02 & 3.37 & 7.35 & 1.63 & 7.69 & 0.12 \\
\hline $\mathrm{T} 1 \mathrm{~A}$ & 4.33 & 0.14 & 3.97 & 2.57 & 4.07 & 1.86 & ${ }^{3} 4.41$ & 0.10 \\
\hline $\mathrm{T} 1 \mathrm{D}$ & ${ }^{1} 4.11$ & 0.11 & 3.61 & 4.55 & 4.04 & 0.64 & 4.08 & 0.08 \\
\hline $\mathrm{T} 2$ & 7.88 & 0.23 & 7.25 & 2.74 & 7.82 & 0.26 & 7.76 & 0.21 \\
\hline T2 A & 4.50 & 0.18 & 3.97 & 2.94 & 4.40 & 0.56 & 4.42 & 0.17 \\
\hline $\mathrm{T} 2 \mathrm{D}$ & 23.81 & 0.12 & 3.54 & 2.25 & 3.8 & $* 0.17$ & ${ }^{4} 3.76$ & 0.09 \\
\hline
\end{tabular}

$\sigma=$ one standard deviation; $\# \sigma=$ number of standard deviations. In the \# $\sigma$ columns, the sign * before a number means the number of $\sigma$ 's above the mean value of $a a$. No sign before a number means the number of $\sigma$ 's below the mean value of $a a .{ }^{1}$ Average during days $1-26 .{ }^{2}$ Average during days $1-24 .{ }^{3}$ Average during days $28-40$. ${ }^{4}$ Average during days $26-40$

First column: type of cycle. Second and third columns: means of the time intervals between day 1 to one day before the Aa minimum and their standard deviations, respectively. Fourth and fifth columns: Aa minima day value and the number of standard deviations of these values with respect to the mean (according to the third column). Sixth and seventh columns: Aa value at new Moon and the number of standard deviations of these values with respect to the mean (according to the third column). Eighth and ninth columns: Aa mean value for days 29-40 and their corresponding standard deviations, respectively. This time span included the full Moon

et al. 2012). Our satellite travels through the tail of the Earth's magnetosphere for 4 or 5 days (Hapgood 2007), and the Moon enters the magnetotail typically $2-2.5$ days before the full Moon. Therefore, at 3-5 days before the full Moon, when the $A a$ index drops are observed, the Moon is located in the magnetosheath. However, even when the Moon is magnetically connected to the Earth in the magnetotail, the spatial scale of the Moon-related perturbed plasma is at most several lunar radii across the field lines. Therefore, the perturbations are local and seem incapable of affecting the global geomagnetic activity, as the magnetosphere is several tens of Earth radii at the lunar distance. In addition, the $A a$ drop occurred only when the Moon enters the magnetosphere and not at its exit. Furthermore, the lunar tidal effects also contribute to the $A a$ perturbation mainly during the new/ full Moon (Yamazaki 2014). However, we cannot see how the tidal effect could explain the $A a$ drop only around the full Moon and not around the new Moon, when a similar behavior could be expected. Apparently, we cannot explain the $A a$ drops through lunar magnetic connection to the magnetosphere or through tidal effects on the ionosphere. Further detailed studies of both the solar and lunar effect on the geomagnetic activity at monthly timescales should be conducted, but this is out of the scope of this study.

During the descending phase, the high-speed streams make the largest contributions to $A a$ and IMF (Richardson et al. 2002). The presence of high-speed streams during Type 2 cycles should produce a stable interaction with the Moon, because it is during this phase that we observed the smallest $A a$ values and changes. Usually during Type 1 cycles, the Moon should have a larger interaction with high-speed streams and the magnetosphere compared to that during Type 2 cycles.

Analyzing the $A a$ index data on monthly timescales from 1868 to the present (Aa activity cycles $11-24$ ), we concluded that:

- There are solar- and lunar-associated periodicities in the $A a$ index.

- There are statistically significant $A a$ minima values a few days before the full Moon.

- There are high $A a$ values during the new Moon.

- When considering all the cycles, it is clear that the deepest $A a$ minima occurred during the descending phase, but when the cycles are separated into Type 1 and 2 (according to the direction of the interplanetary magnetic field), we noticed that the $A a$ minima in all the cycles came from the contribution of the Type 1 descending phase.

- The Type 2 descending phase cycles presented the smallest $A a$ index values and smaller changes compared to Type 1 cases. This behavior implies that during Type 1 cycles there are larger $A a$ perturbations than during Type 2 cycles.

- It is very likely that the mechanisms through which the Sun and Moon affect the geomagnetic activity on monthly timescales depend on several factors. In this study, we have identified the following: (a) the Moon phases (new and full Moon), (b) the phase of the solar cycle (ascending or descending), and (c) the direction of the interplanetary magnetic field (away or toward the Sun). 


\section{Additional file}

Additional file 1. Time series of the aa index and moon luminosity from January 1868 through January 2015.

\section{Authors' contributions}

BM wrote the main manuscript and performed the data analysis. MP wrote the methodology and performed the data analysis and drew the figures. LXG obtained all the data and arranged it to perform the analysis. All authors read and approved the final manuscript.

\section{Authors' information}

B. Mendoza is currently the Coordinator of The Earth Sciences Bachelor's Degree at the Universidad Nacional Autónoma de México (UNAM) and has the maximum level in the Mexican National Research System. She is also a member of the Space Sciences Department at UNAM. Her main field of research is Solar Physics and Sun-Earth relationships, in particular space weather impacts on Earth's climate and biota. M. Pazos has a Ph.D. degree in Space Physics from the Universidad Nacional Autónoma de México, and her main field of research is Sun-Climate relationships. L. X. González is member of the Mexican Space Weather Service in the Instituto de Geofisica of the Universidad Nacional Autónoma de México (UNAM). He belongs to the Mexican National Research System and his main field of research is Solar Physics, Space Weather and Cosmic Ray Detectors.

\section{Author details}

${ }^{1}$ Instituto de Geofísica, Universidad Nacional Autónoma de México, 04510 Mexico City, Mexico. ${ }^{2}$ SCiESMEX, Instituto de Geofísica, Unidad Michoacán, Universidad Nacional Autónoma de México, 58190 Morelia, Michoacán, Mexico.

\section{Acknowledgements}

M. Pazos and L. X. González acknowledge the support of grants CB2012/180727-CONACyT and CONACyT/AEM-2014-01-247722, respectively.

\section{Competing interests}

The authors declare that they have no competing interests.

\section{Availability of data and materials}

The dataset supporting the conclusions of this study is included in an additional file.

Received: 20 April 2016 Accepted: 17 November 2016

Published online: 01 December 2016
Campbell WH (1997) Introduction to geomagnetic fields. Cambridge University Press, UK

Grinsted A, Moore J, Jevrejera S (2004) Application of the cross wavelet transform and wavelet coherence to geophysical time series. Nonlinear Process Geophys 11:561-566

Gulyaev RA, Gulyaeva TL (1992) Alternative non gravity mechanisms of lunar influence upon the biological processes. Biofizika 37:541-545

Halekas JS, Poppe AR, Farrell WM, Delory GT, Angelopoulos V, McFadden JP, Bonnell Glassmeier KH, Plaschke F, Roux A, Ergun RE (2012) Lunar precursor effects in the solar wind and terrestrial magnetosphere. J Geophys Res 117:A05101

Hapgood M (2007) Modelling long-term trends in lunar exposure to the Earth's Plasmasheet. Ann Geophys 25:2037-2044

Mayaud P-R (1972) The aa indices: A 100-year series characterizing magnetic activity. J Geophys Res 77:6870-6874

Mendoza B, Velasco V (2009) High-latitude methane sulphonic acid variability and solar activity: the role of the total solar irradiance. J Atm Solar-Terr Phys 71:33-40

Michel FC, Dessler Al, Walters GA (1964) A search for correlations between Kp and lunar phases. J Geophys Res 69:4177-4181

Mitchell DL, Halekas JS, Lin RP, Frey S, Hood LL, Acuna MH, Binder A (2008) Global mapping of lunar crustal magnetic fields by Lunar Prospector. Icarus 194:401-409

Poppe A, Horányi M (2010) Simulations of the photoelectron sheath and dust levitation on the lunar surface. J Geophys Res 115:A08106

Richardson IG, Cane HV, Cliver EW (2002) Sources of geomagnetic activity during nearly three solar cycles (1972-2000). J Geophys Res 107(A8):SSH1-SSH13

Russell CT, Mulligan T (1995) The 22-year variation of geomagnetic activity: implications for the polar magnetic field of the Sun. Geophys Res Lett 22:3287-3288

Saito Y, Nishino MN, Fujimoto M, Yamamoto T, Yokota S, Tsunakawa H, Shibuya H, Matsushima M, Shimizu H, Takahashi F (2012) Simultaneous observation of the electron acceleration and ion deceleration over lunar magnetic anomalies. Earth Planets Space 64:83-92. doi:10.5047/ eps.2011.07.011

Stolov HLO, Cameron AGW (1964) Variations of geomagnetic activity with lunar phases. J Geophys Res 69:4975

Střeštík J (1998) Spectrum of geomagnetic activity in the period range $5 \pm 60$ days: possible lunar influences. Ann Geophys 16:804-811

Torrence C, Compo G (1998) A practical guide to wavelet analysis. Bull Am Meteorol Soc 79:61-78

Yamazaki Y (2014) Solar and lunar ionospheric electrodynamic effects during stratospheric sudden warmings. JASTP 119:138-146

Yamazaki Y, Richmond AD (2013) A theory of ionospheric response to upwardpropagating tides: electrodynamic effects and tidal mixing effects. J Geophys Res Space Phys 118:5891-5905

\section{References}

Akimov LA, Dyatel NP (2012) Influence of the Moon on the Earth's magnetosphere at various phases of a solar activity cycle. Kinemat Phys Celest Bodies 28:39-42

\section{Submit your manuscript to a SpringerOpen ${ }^{\circ}$ journal and benefit from:}

- Convenient online submission

- Rigorous peer review

Immediate publication on acceptance

- Open access: articles freely available online

- High visibility within the field

- Retaining the copyright to your article

Submit your next manuscript at springeropen.com 